\title{
Design of permanent magnets to chaoize doubly salient permanent magnet motors for electric compaction
}

\author{
K. T. Chau and Zheng Wang ${ }^{\text {a) }}$ \\ Department of Electrical and Electronic Engineering, The University of Hong Kong, Hong Kong, China
}

(Presented on 3 November 2005; published online 19 April 2006)

\begin{abstract}
This article proposes and implements a class of chaotic motors for electric compaction. The key is to develop a design approach for the permanent magnets (PMs) of doubly salient PM (DSPM) motors in such a way that chaotic motion can be naturally produced. The bifurcation diagram is employed to derive the threshold of chaoization in terms of PM flux, while the corresponding phase-plane trajectories are used to characterize the chaotic motion. A practical three-phase 12/8-pole DSPM motor is used for exemplification. The proposed chaotic motor is critically assessed for application to a vibratory soil compactor, which is proven to offer better compaction performance than its counterparts. Both computer simulation and experimental results are given to illustrate the proposed chaotic motor. (C) 2006 American Institute of Physics.
\end{abstract}

[DOI: $10.1063 / 1.2165783$ ]

\section{INTRODUCTION}

Recently, a class of brushless motors, namely, the doubly salient permanent magnet (DSPM) motor, has been introduced. ${ }^{1}$ The DSPM motor combines the advantages of both the switched reluctance motor and the PM brushless motor, hence achieving robust structure, high power density, high efficiency, high reliability, and maintenance-free operation. Thus, it is attractive for high-performance applications such as electric vehicle propulsion and wind power generation.

Starting from the 1990s, many research activities on chaos in motors have been carried out. Most of them are based on the identification, avoidance, and stabilization of chaos in various motors. Recently, the design of PMs to avoid chaos in DSPM motors ${ }^{2}$ has also been discussed. Rather than negatively avoiding the occurrence of chaos in motors, a positive idea is to utilize the chaotic motor for some niche applications. In Ref. 3, chaotic motion was firstly applied to soil compaction, and was experimentally proven to exhibit better compaction performance. However, this chaotic motion is generated by a complicated mechanical system, which suffers from the problems of bulky size and inflexible design.

The purpose of this article is to explore a design approach for chaoization of DSPM motors, and to positively utilize the resulting chaotic motion for electric compaction. Hence, this electrically chaotic compactor can take definite advantages of simplicity, compactness and flexibility over its mechanically chaotic counterpart.

\section{SYSTEM DYNAMICS}

A three-phase 12/8-pole DSPM motor is adopted for exemplification. As shown in Fig. 1, it consists of threephase armature windings in the stator, four pieces of PM material located in the stator, twelve salient poles in the stator, and eight salient poles in the rotor. With rotor skewing, the PM flux can be expressed as

$$
\psi_{\mathrm{pm} x}=\psi_{\mathrm{pm} 0}+\psi_{\mathrm{pm} 1} \cos \left(n_{p} \theta-2 \pi k / 3\right),
$$

where both $x=a, b, c$ and $k=0,1,2$ correspond to the phases $\mathrm{A}, \mathrm{B}, \mathrm{C}$, respectively, $\psi_{\mathrm{pm} 0}$ and $\psi_{\mathrm{pm} 1}$ are the average and amplitude of PM flux variations, respectively, $n_{p}$ is the number of pole pairs, and $\theta$ is the spatial angle with reference to the aligned position of phase A. The mutual inductances of the windings are negligible, whereas the self-inductances can be written as

$$
L_{x x}=L_{0}+L_{1} \cos \left(n_{p} \theta-2 \pi k / 3\right),
$$

where $L_{0}$ and $L_{1}$ are the average and amplitude of selfinductance variations, respectively. Hence, the dynamic equations of this DSPM motor are given by

$$
\begin{aligned}
& \frac{d i_{x}}{d t}=\frac{1}{L_{x x}}\left(u_{x}-\frac{d L_{x x}}{d t} i_{x}-R i_{x}-\frac{d \psi_{\mathrm{pm} x}}{d t}\right), \\
& \frac{d \omega}{d t}=\frac{1}{J}\left(T_{e}-T_{l}-B \omega\right),
\end{aligned}
$$

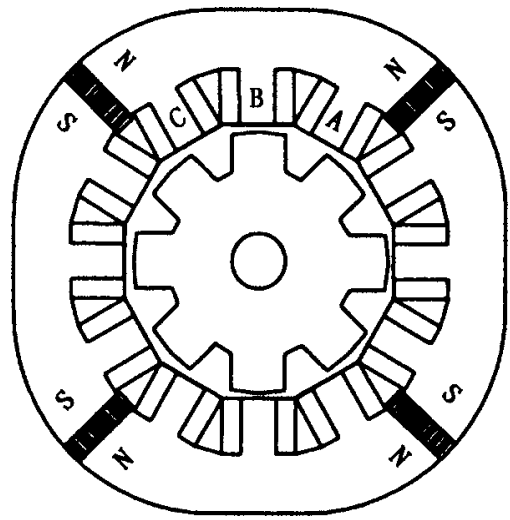

FIG. 1. Configuration of three-phase 12/8-pole DSPM motor.

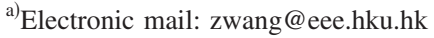




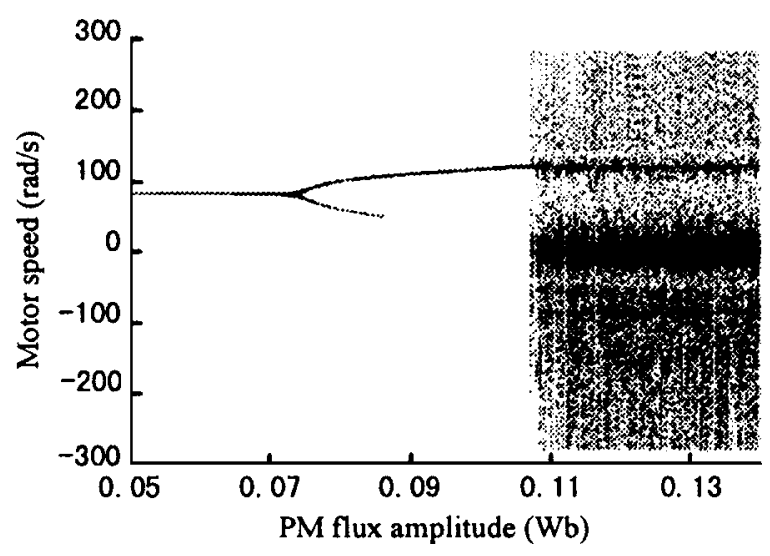

FIG. 2. Motor speed bifurcation diagram.

$$
T_{e}=\frac{1}{\omega_{x=a, b, c}} \sum_{d}\left(\frac{d L_{x x} i_{x}}{d t}+\frac{d \psi_{\mathrm{pm} x}}{d t}\right) i_{x}
$$

where $i_{x}$ is the phase current, $u_{x}$ is the phase voltage, $R$ is the winding resistance, $\omega$ is the motor speed, $T_{e}$ is the electromagnetic torque, $T_{l}$ is the load torque, $B$ is the viscous coefficient, and $J$ is the moment of inertia.

\section{PM DESIGN FOR CHAOIZATION}

The system model given by Eqs. (1)-(5) illustrates that the variation of PM flux is an important parameter contributing to system dynamics. Thus, the design of PMs, mathematically the value of $\psi_{\mathrm{pm} 1}$, is used for chaoization of the DSPM motor.

By adopting practical data of the DSPM motor, namely, $n_{p}=4, L_{0}=90 \mathrm{mH}, L_{1}=45 \mathrm{mH}, U_{x}=35.4 \mathrm{~V}, R=2.5 \Omega, B$ $=4.584 \times 10^{-4} \mathrm{~N} \mathrm{~m} \mathrm{~s}$, and $J=1 \times 10^{-4} \mathrm{~kg} \mathrm{~m}^{2}$, the bifurcation diagram of $\omega$ with respect to $\psi_{\mathrm{pm} 1}$ can be obtained. As shown in Fig. 2, it can be seen that the motor speed changes from normal (constant-speed) operation to subharmonic operation at $\psi_{\mathrm{pm} 1}=0.073 \mathrm{~Wb}$, and then bifurcates to chaotic operation at $\psi_{\mathrm{pm} 1}=0.108 \mathrm{~Wb}$ (so-called the threshold of chaoization). When selecting $\psi_{\mathrm{pm} 1}=0.07 \mathrm{~Wb}$, the simulated wave forms of motor speed and phase current are shown in Fig. 3, which are actually the normal operating wave forms. On the other hand, when selecting $\psi_{\mathrm{pm} 1}=0.12 \mathrm{~Wb}$, the simulated wave forms of motor speed and phase current are shown in Fig. 4. It can be found that these wave forms exhibit a well-known chaotic behavior of a random-like nature. Moreover, the corresponding phase-plane trajectories $\left(i_{x}\right.$ versus $\left.\omega\right)$ are shown
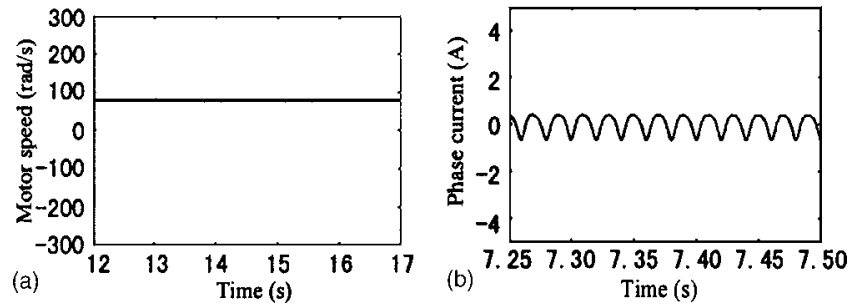

FIG. 3. Simulated normal operating wave forms. (a) Motor speed. (b) Phase current.
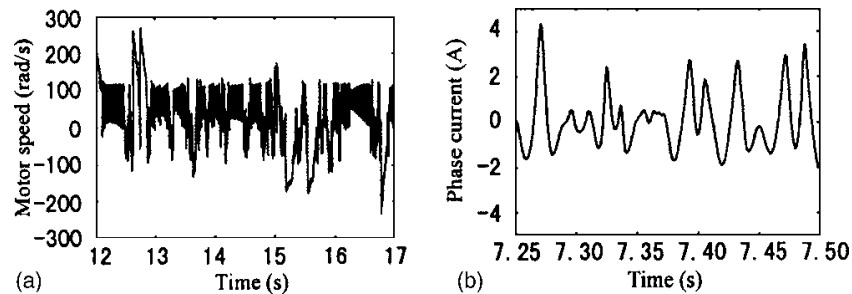

FIG. 4. Simulated chaotic operating wave forms. (a) Motor speed. (b) Phase current.

in Figs. 5(a) and 5(b), respectively, confirming that the chaotic trajectory exhibits another well-known behavior of a bounded nature.

\section{APPLICATION TO COMPACTION}

As soil is constituted by different sizes and different materials of granules, its natural frequencies are widely dispersed. Thus. constant-speed compactor can only provide effective compaction for an ideal type of soil which has a single natural frequency. Since chaos is characterized by exhibiting a wide frequency spectrum, it is anticipated that chaotic motion can provide effective compaction for realistic soil which has a wide range of natural frequencies. Thus, the proposed chaotic motor is applied to drive a vibratory compactor. Figure 6 shows the model of this compactor, ${ }^{4}$ in which the interaction between the framework and the vibratory drum is represented by the stiffness coefficient $K_{f}$ $=5.25 \times 10^{6} \mathrm{~N} / \mathrm{m}$ and damping coefficient $C_{f}=2.63$ $\times 10^{3} \mathrm{~N} \mathrm{~s} / \mathrm{m}$, and the interaction between the soil and the vibratory drum is described by $K_{s}=3.52 \times 10^{6}-5.87$ $\times 10^{7} \mathrm{~N} / \mathrm{m}$ and $C_{s}=8.31 \times 10^{3}-3.39 \times 10^{4} \mathrm{~N} \mathrm{~s} / \mathrm{m}$.

In order to critically assess the compaction performance of the proposed chaotic compactor as compared with the conventional constant-speed compactor, they are required to compact a set of soils with the natural frequencies evenly distributed between 235 and $1000 \mathrm{~Hz}$. The comparison is based on three well-accepted compaction performance indicators; namely, the displacement of compactor plate, the vertical exciting force exerted onto the soil, and the average compaction energy density. ${ }^{4}$ The constant-speed operation is produced by using $\psi_{\mathrm{pm} 1}=0.07 \mathrm{~Wb}$, while the chaotic operation is generated by using $\psi_{\mathrm{pm} 1}=0.12 \mathrm{~Wb}$. Figures $7-9$ show their comparisons in terms of these three indicators, where the crosses represent the individual effects, and the horizontal line is their averaged value. As expected, all of them confirm that the proposed chaotic compactor provides much better compaction performance than the constant-speed one.
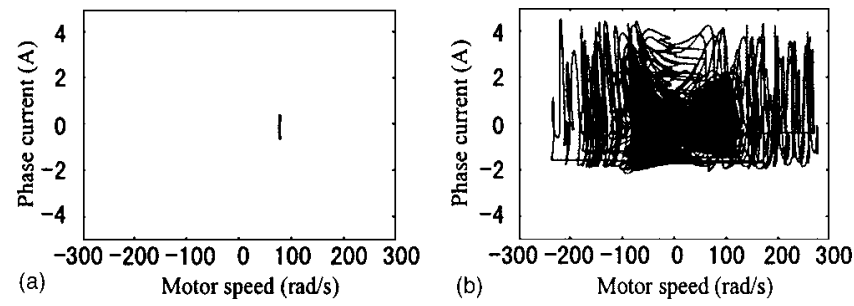

FIG. 5. Simulated trajectories. (a) Normal operation. (b) Chaotic operation. 


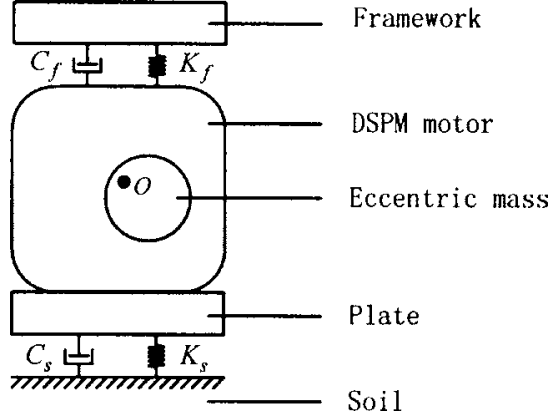

FIG. 6. Configuration of vibratory compactor.
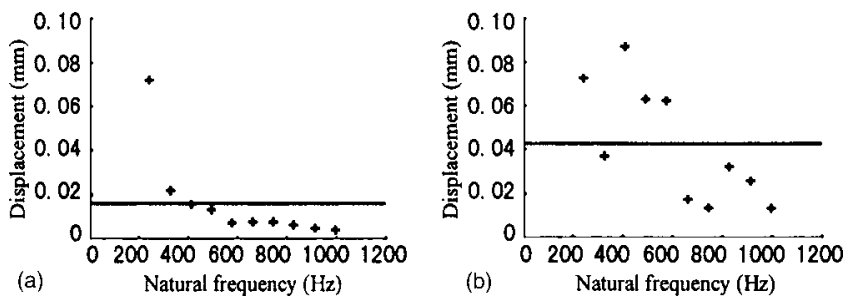

FIG. 7. Displacement. (a) Normal operation. (b) Chaotic operation.
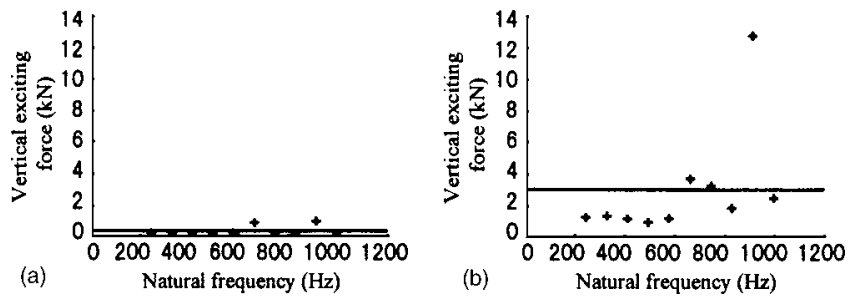

FIG. 8. Vertical exciting force. (a) Normal operation. (b) Chaotic operation
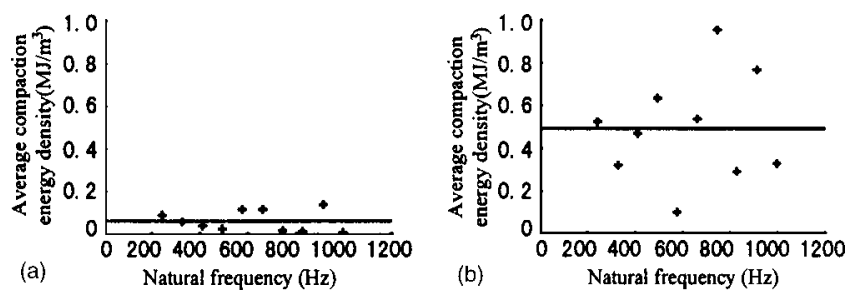

FIG. 9. Average compaction energy density. (a) Normal operation. (b) Chaotic operation.

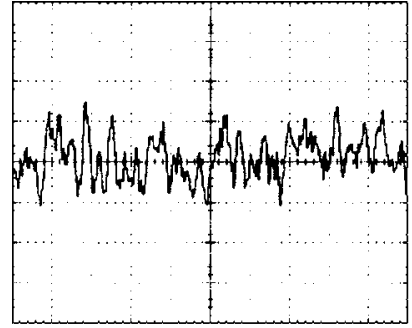

(a)

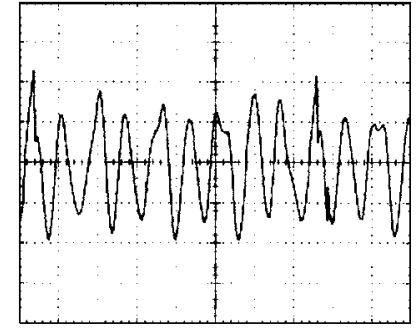

(b)
FIG. 10. Measured chaotic operating wave forms. (a) Motor speed (200 rad/s/div, $500 \mathrm{~ms} /$ div). (b) Phase current (2 A/div, $25 \mathrm{~ms} / \mathrm{div})$.

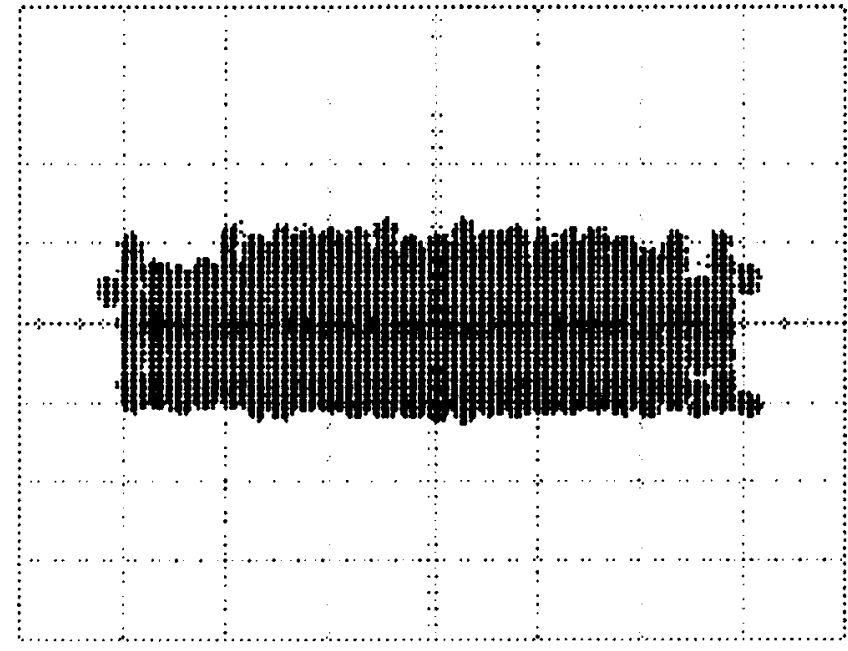

FIG. 11. Measured chaotic operating trajectory of phase current versus motor speed (4 A/div, $80 \mathrm{rad} / \mathrm{s} / \mathrm{div})$.

\section{EXPERIMENTAL VERIFICATION}

In order to further verify the proposed design approach, the newly designed DSPM motor with $\psi_{\mathrm{pm} 1}=0.12 \mathrm{~Wb}$ is prototyped and tested. Figure 10 shows the measured chaotic operating wave forms of motor speed and phase current, and Fig. 11 shows the corresponding phase-plane trajectory. It can seen that they quite agree with the simulation results, and exhibit the expected random-like and bounded nature. Moreover, by calculating the maximum Lyapunov exponent of the measured time series, it is found to be $4.856>0$, hence mathematically confirming that the proposed motor provides chaotic motion.

\section{CONCLUSIONS}

In this article, a class of chaotic DSPM motors has been proposed. The bifurcation diagram has been employed to derive the threshold of chaoization in terms of PM flux, while the corresponding phase-plane trajectories are used to characterize the chaotic motion. The proposed chaotic motor has been applied for soil compaction, hence confirming that it offers better compaction performance than the constantspeed one. Experimental results have been given to verify the computer simulation.

\section{ACKNOWLEDGMENT}

This work was supported by a grant from the Research Grants Council of Hong Kong, China (Project No.HKU7154/ 04E).

${ }^{1}$ M. Cheng, K. T. Chau, and C. C. Chan, IEEE Trans. Magn. 37, 3012 (2001).

${ }^{2}$ Y. Gao and K. T. Chau, IEEE Trans. Magn. 40, 3048 (2004).

${ }^{3}$ Y. J. Long, Y. Yang, and C. L. Wang, Engineering Science 2, 76 (2000).

${ }^{4}$ D. T. Tran and T. Muro, J. Terramech. 41, 1 (2004). 